\title{
Impact of Insomnia on Optimism-A Predictor Factor Among Young Adults in Indian Context
}

\author{
Manisha Choudhury ${ }^{1 *}$, Aprajita Jayaswal ${ }^{2}$ and Parul $^{3}$ \\ ${ }^{1}$ Department of Audiology and Speech Language Pathology, Amity University Haryana, India \\ ${ }^{2}$ Department of Psychiatric Consultation and Counseling, Assistant Professor, Amity University Haryana, India \\ ${ }^{3}$ Clinical Psychologist, India
}

*Corresponding author: Manisha Choudhury, Department of Audiology and Speech Language Pathology, Amity University Haryana, India

\begin{abstract}
Recent research studies have revealed that inadequate quantity of sleep cycle and deprived sleep quality, are one of the most common emerging issues which are affecting the personality and attitude traits of an individual. There are significant research studies conducted which indicates optimism and self-esteem are the core salient features for maintaining a good health.

Aim of the study: There is a dearth of knowledge regarding the possible determinants of positive optimism characteristics among young adults. Therefore, the present study was conducted to examine the relationship of optimism with insomnia symptoms among young adults.
\end{abstract}

Method: Sleep parameters and optimism were assessed by administering standardized questionnaire(s) among a sample of 92 young adults aged between 17 and 26 years studying in University.

Results: Statistical analysis shows significant correlation between optimism scale and sleep cycle, the participants exhibiting higher score of insomnia tend to show lower score on optimism scale.

Conclusion: The result provides preliminary input on risk factors for insomnia and effect on optimism, as adequate amount of sleep has a strong relationship with optimism leading to a more confident and improved quality of life.

Keywords: Insomnia Symptoms; Sleep Duration; Optimism

\section{Introduction}

Emerging research supports the findings that nocturnal sleep is one of the most important aspect of our life for maintaining a sound physical and mental health. This area is one of the emerging areas in the field of psychology which has been immensely studied. Various research studies indicate that sleeping less than 7 hours as well as sleeping more than 8 hours is closely linked to an increased susceptibility to a broad range of physical and psychological health problems, such as ranging from poor vigilance and memory to reduced mental and physical reaction times, reduced motivation, depression, insomnia, metabolic abnormalities, obesity, immune impairment, and even a greater risk of coronary heart disease and even cancer [1,2]. Longitudinal evidence suggests that, insomnia is most common co-morbid condition seen with mood, anxiety and predates the onset of low optimistic attitude within an individual
[3]. Numerous research studies also reveal significant results which indicates that sleep deprivation among young adults tends to interfere with the mechanism, which is responsible for regulating personality characteristics including optimism, with increased risk for anxiety, negative mood, impulsivity, and inability to cope up with social stresses [4-8].

\section{Neurobiology of Sleep}

The circadian rhythm i.e. the sleep-wake cycle is controlled by the suprachiasmatic nucleus of hypothalamus [9]. The inhibitory projections of ventrolateral preoptic nucleus (VLPO) of the hypothalamus to the tuberomammillary nucleus (TMN), the dorsal and median raphe nucleus and the locus coeruleus, the cholinergic basal forebrain, the pedunculopontine thalamic 
nucleus (PPT) and lateral dorsal thalamic nucleus (LDT) functions as a switch promoting sleep [10]. The orexinergic neurons of lateral hypothalamic area (LHA) promote wakefulness and the inhibitory effect of VLPO promotes sleep [10].

\section{Models of Insomnia}

There are various models which define the foundation of insomnia explaining the various factors that are responsible for related sleep disturbances. One such model is The Diathesis Stress Model or the 3-P model (predisposing, precipitating and perpetuating) factors given by Spielman and colleagues (2011) mentioned the role of the three P's in development and maintenance of insomnia. The insomnia symptoms worsen in an attempt to relieve it hence the model focuses on behavioral pattern [11]. For example, an attempt to compensate for reduced sleep by spending increased time in bed, may worsen insomnia unintentionally. The classical conditioning principles proposed by Bootzin (1972) is the basis of the stimulus control model which states that the person with insomnia becomes conditioned to sleep and bed environment as a stimulus for wakefulness instead of sleep. The therapy attempts to decondition to this stimulus by limiting activities like lying awake, watching TV in bed and ensuring bed is used for sleep only [12]. According to Cognitive Model of insomnia thoughts, (worry about poor sleep and its daytime effects) and associated feeling interferes with the sleep [13]. Study has suggested that scanning of the internal and environment threat signals, and the safety behaviours to increase sleep and minimize the outcome of insomnia ,worsens insomnia [13] whereas, the role of selective attention to sleeprelated indicators in the development and maintenance of insomnia is as per the basis of the Psychobiological Inhibition Model [14]. As per Diagnostic Guidelines of Insomnia (ICD-10, 2004) the following are essential clinical features for a definite diagnosis [15].

a) The complaint is either of difficulty falling asleep or maintaining sleep or of poor quality of sleep.

b) The sleep disturbance has occurred at least three times per week for at least 1 month.

c) There is preoccupation with the sleeplessness and excessive concern over its consequences at night and during the day.

d) The unsatisfactory quantity and/or quality of sleep either causes marked distress or interferes with ordinary activities in daily living.

e) The unsatisfactory quantity and /or quality of sleep is the patients only complain.

Compared to the research studies conducted on consequences of poor sleep, there is inadequate evidence regarding the association between sleep duration with positive individual characteristics. According to the data published by National Sleep Foundation (2002) revealed that individuals who had an average sleeping duration of $7-8$ hours reported to have better mental satisfaction with life compared to the other group of individuals who had a sleeping duration of less than 6 hours a night [16]. Fredriksen (1994) conducted another similar kind of study in which the results obtained were evident indicating that longer the sleep duration higher is the self-esteem in adolescents [17]. Various other studies revealed a strong association between ample sleep duration and short sleep onset latency resulting in higher optimism in children [3]. Another experimental study was administered to examine the effect of insomnia and the results indicated that sleep deprivation leads to gradual degradation of self-reported optimism and poor social interaction in young adults, which suggested that sleep duration is a determinant factor for the initiation of positive personality characteristics in an individual [18]. Most of the research studies say that lack of sleep may lead to co-morbid condition which could be anxiety and depressive symptoms and also lack of optimism. In the present study, we therefore tested associations of sleep parameters with optimism among university students aged 17 to 26 years. In particular, the study was conducted to examine the relation between symptoms of insomnia and optimism. Simultaneously, also aimed to evaluate whether individuals with an average sleep duration (between 7 and 8 hours/day) are more optimistic compared to individuals who sleep less than 7 hours or, conversely, more than 8 hours per day [19].

\section{Method \\ Participants}

A total of 92 subjects were selected using purposive sampling based on their encouragement to participate in the present study from the university. The participants were divided into

a) Group 0:63 (aged 17-21 years)

b) Group 1:29 (aged 22-26 years)

Individuals who fulfilled the criteria of insomnia with the minimum age of 18 years were included in the study. On the other hand, individuals who were suffering from other psychiatric illness or co morbid conditions were excluded from the study. All the subjects were explained in detail regarding the purpose of the study. Primarily, the mother tongue of all the participants was Hindi simultaneously had a good knowledge of English. Informed consent and personal information were taken from the participant.

\section{Materials Used}

Two questionnaires were administered:

a) Pittsburgh Sleep quality Index (PSQI) which consisted of 19 individual items, creating 7 components. Higher the score on Pittsburgh quality index, the lower is the sleep quality and vice versa [19].

b) Optimism sleeping Index (OSI) to measure the five facets of Optimism. There are 60 items in the test which are in 5-point Likert system. All the questions were closed set task with 3-point rating scale i.e., yes or no and not sure [20].

The data collected was analyzed using SPSS (version 17, IBM Corporation, Bengaluru, India), along with percentages of the study subjects, with respect to a particular response. The percentages and proportions of different categories of questionnaires were used to analyze the data. 


\section{Results and Discussion}

The aim of the study was to find out the relationship between insomnia and five facets of Optimism among University students. On statistical analysis, the correlation (Table 1) between Pittsburgh Sleep Quality Index and Optimism Index was found to be: $(r=-.342$, $\mathrm{P}<.01)$. Therefore, the findings reveal that there is a negative correlation between Sleeping Difficulties and Optimism which means higher level of optimism is related to better Sleep Quality and more sleeping difficulties relate to lower level of optimism. Further, the data analysed on optimism five facets Positive Emotions (P_E), Engagement (E_M), Meaningfulness(M_F), Relationships(R_S) and
Accomplishment(A_C) also provides negative correlation is found again between Sleep Difficulties using PSIQ and each Optimism Facet using OSI (Table 1). Extensive research study on optimism; which is an major aspect of positive personality is important as it is considered one of core platform for the development subjective wellbeing and health [21].A prospective review study was conducted over a 9 year follow-up period which showed a protective effect of dispositional optimism against various cardiovascular mortality in old age controlling the initial health status [22].Considering the literature current study also revealed similar result findings among the university students. The detailed description are as follows:

Table 1: Variation in Correlation between Optimism, its facet \& Pittsburgh Sleep Quality Index with different age groups.

\begin{tabular}{|c|c|c|}
\hline \multirow{2}{*}{ Variable } & Correlation (n=92 & \multicolumn{2}{|c|}{ Norrelation Age Group } \\
\cline { 2 - 3 } & -0.342 & -0.354 \\
\hline Total (Optimism Index) & -0.304 & -0.338 \\
\hline Positive Emotions (P_E) & -0.241 & -0.273 \\
\hline Engagement (E_M) & -0.289 & -0.251 \\
\hline Relationship (M_F) & -0.258 & -0.196 \\
\hline Meaningfulness (R_S) & -0.289 & -0.298 \\
\hline Accomplishment (A_C) & 1 & -0.3 \\
\hline Pittsburgh Sleep Quality Index (PSQI) & -0.2 & 1 \\
\hline
\end{tabular}

a) Positive Emotions and PSIQ: On statistical analysis, the correlation between Positive Emotions and Pittsburgh Sleep Quality Index was found to be: $(\mathrm{r}=-.304, \mathrm{P}<.01)$ as shown in (Figure 1). This indicates that there is a negative correlation between Sleeping Difficulties \& Positive Emotions. This implies the inference that good quality sleep is related to higher value of positive emotions and vice versa.

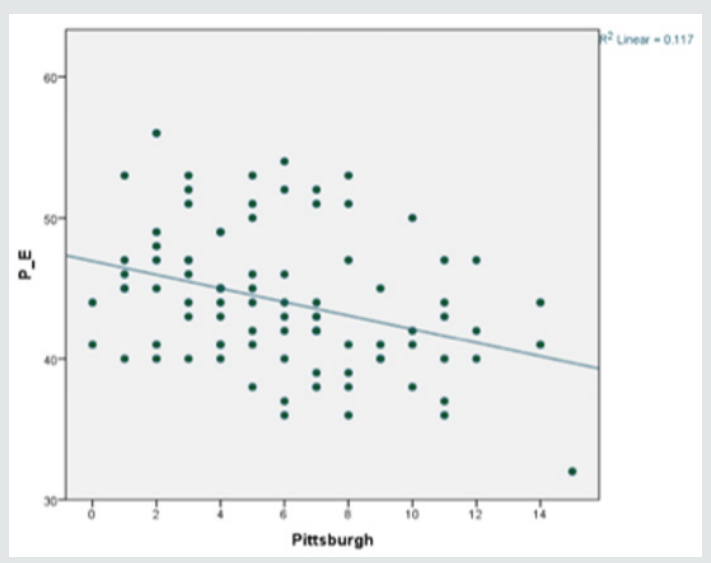

Figure 1: Graph representing relationship between Sleep Quality Index and Positive Emotions.

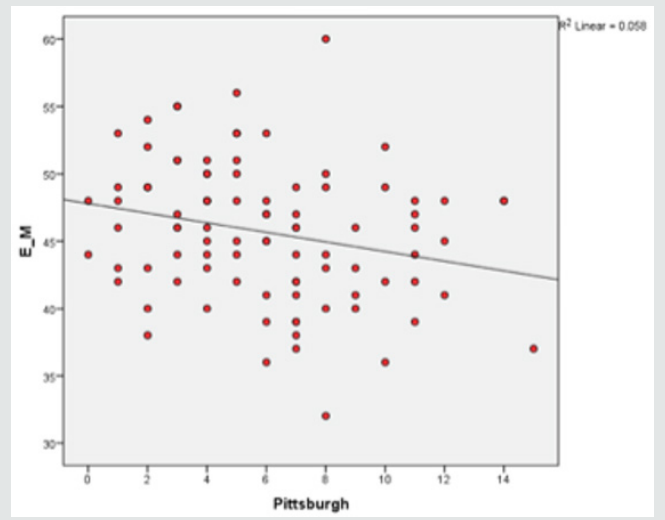

Figure 2: Graph representing relationship between Sleep Quality Index and Engagement. 
b) Engagement and PSIQ: The relationship between Engagement and Pittsburgh sleep quality index is ( $\mathrm{r}=-.241, \mathrm{P}$ $<.05)$ which implies a negative correlation between Sleeping Difficulties and Engagement also (Figure 2). A higher value of engagement is seen in students with lower Pittsburgh sleep quality index score as compared to those with higher Pittsburgh score. This implies the same inference as with above facet i.e. good quality sleep relates to good engagement score and Sleeping Difficulties relate to low engagement score/bad engagement skills.

c) Relationship and PSIQ: The correlation value between Relationship and sleep quality Index: $(\mathrm{r}=-.289, \mathrm{P}<.01)$. A negative correlation value also indicates that this facet of optimism also relates to a higher value corresponding to lower values on Pittsburgh Sleep Quality Index (Figure 3). This again confirms our inference that more Sleeping Difficulties are related to lower inter-personal Relationship skills. A good quality sleep is related to a higher level of Relationship values in students. There are similar supporting studies which shows that people with lower social network and relationships tend to exhibit poor sleep. Researchers pointed out the importance of relationship support on health which indicated that higher level of non-reciprocity in social interaction, the higher level of sleep problems, depression and lower level of physical and mental health [23].

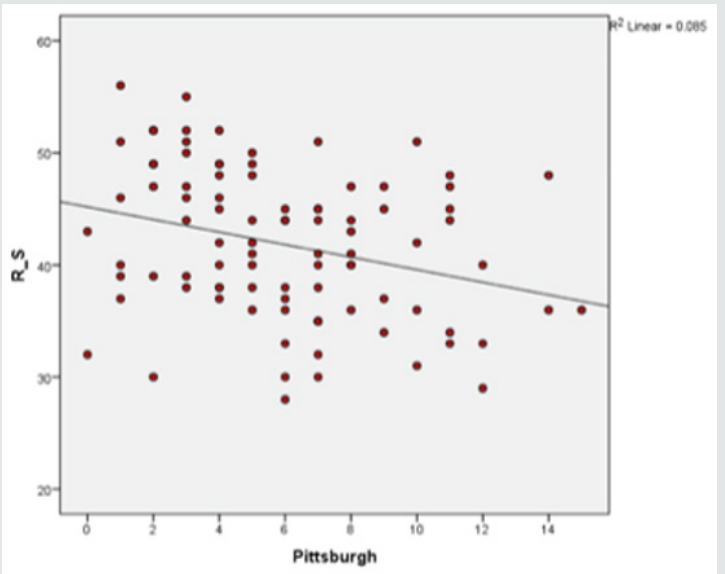

Figure 3: Graph representing relationship between Sleep Quality Index and Relationship.

d) Meaningfulness and PSIQ: The fourth facet of optimism is meaningfulness which correlates as: $(r=-.258, P<.05)$ with the Pittsburgh score. This value also suggests that a good meaningfulness score is seen in students with good quality sleep as compared to those with sleep difficulties (Figure 4). Haack And Mullington [24] demonstrated that sleep deprivation resulted in a gradual reduction of self-reported optimism and sociability in young adults, which suggests a causal relation between sleep and positive personality characteristics. There are rich literature studies conducted which shows the relationship between insomnia and physical health [25] wherein optimism on one side has a sleep-enhancing effect; whereas poor sleep constitutes pessimism on the other side [24,25]. Similarly, it's interesting to study that depressive mood fully moderates the first pathway, from optimism to sleep quality, the effects of sleep on optimism are only partially explained by depressive $\operatorname{mood}[26]$.

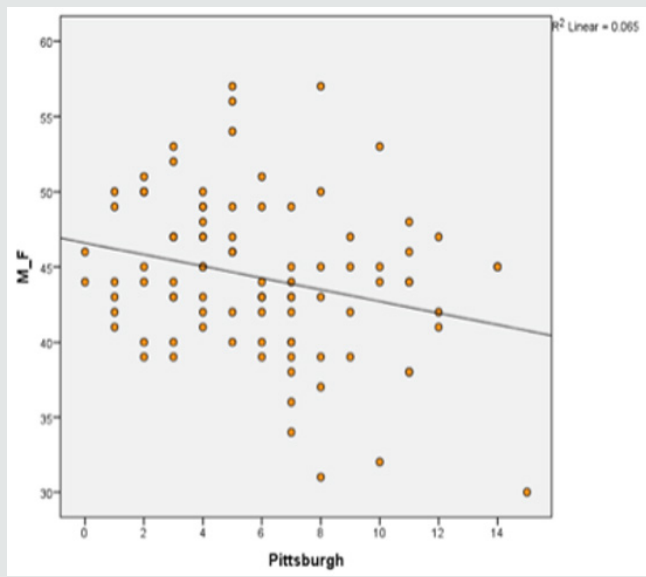

Figure 4: Graph representing relationship between Sleep Quality Index and Meaningfulness. 
e) Accomplishment and PSIQ: The last facet which is accomplishment has a correlation value: $(r=-.289, \mathrm{P}<.01)$ with the Pittsburgh score. This facet is also related to Pittsburgh Sleep Quality Index similarly. Sleeping Difficulties relate to a lower value of accomplishment and good quality sleep relates to a higher value of accomplishment (Figure 5). Robert et al. [27] stated that chronic insomnia is one of the leading causes that can result in poor performances in various aspects, including the interpersonal, somatic and psychological functioning of an individual [27].

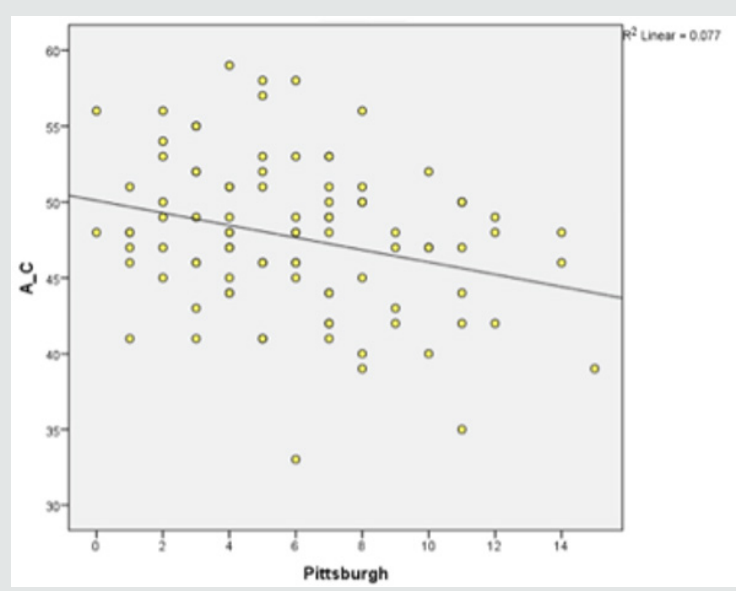

Figure 5: Graph representing relationship between Sleep Quality Index and Accomplishment.

Further, analysis was done to compare the variation in Optimism Index and Pittsburgh Score of participants with variation across the age Group (Table 2). The mean Optimism Index for Age Group 0 \& Age Group 1 (Table 2) were found to be $M(S D)=222.06(18.562) \&$ $M(S D)=226.59(27.514)$. These mean optimism index scores of the students of the two age groups was compared and the variation was found to be just $2.04 \%$, the mean Optimism Index increased by just $2.04 \%$ when moving from lower age group(Group 0) to higher age group(Group 1), which was considered as a marginal difference. This implies in turn reveals that Optimism level was comparatively better in Group 1: "22-26" age group. Thus, this present study also suggests age doesn't have a significant impact on the optimism level of university students. Comparison was also done between the mean Pittsburgh scores of the two age groups. The Mean Pittsburgh Sleep Quality Index Scores for Age Group 0 and Age Group 1 found to be $\mathrm{M}(\mathrm{SD})=6.05(3.333) \& \mathrm{M}(\mathrm{SD})=5.86(3.710)$ which was suggestive marginal/negligible difference. However, there are longitudinal studies conducted on adolescence which revealed that sleep duration decreased with age. Longer sleep duration was concurrently associated with better subjective psychological wellbeing [28] whereas, there was contraindicatory findings which showed that both the age groups had a variation of just 3.14\% in Mean Pittsburgh Index Scores. The Mean Pittsburgh Sleep Quality Index shows a decrease by $3.14 \%$ from lower age group (Group 0) to higher age group (Group1). This finding suggest that Sleep Quality was found marginally better in Group 1 i.e. "22-26" age group which indicates that age group variation among university students doesn't significantly affect their sleep quality which can be related to limited sample size. A similar study was conducted on effect of Optimism and Self-Esteem related to sleep in a large community-Based Sample and results indicated that individuals with insomnia symptoms scored lower on optimism and selfesteem which was largely independent of age and sex, controlling for symptoms of depression and short duration. Thus, the current findings confirm the previous knowledge gained by studies that people with better sleep quality have higher optimism and people with poor sleep quality have lower optimism. Also, that high level of optimism also directly or indirectly results in better sleep quality.

Table 2: Sociodemographic details and Clinical Characteristics of participants $(\mathrm{N}=92)$.

\begin{tabular}{|c|c|c|c|}
\hline \multirow{2}{*}{ Variable } & \multirow{2}{*}{$\begin{array}{c}\text { Sample Size } \\
\text { N=92 }\end{array}$} & \multicolumn{2}{|c|}{ Age group } \\
\cline { 3 - 4 } & “17-21" (n=63) & "22-26" (n=29) \\
\hline \multicolumn{3}{|c|}{ Sociodemographic Characteristics } \\
\hline $\begin{array}{c}\text { Age in years } \\
\text { M(SD) }\end{array}$ & $20.55(2.098)$ & $19.33(1.032)$ & $23.21(1.146)$ \\
\hline $\begin{array}{c}\text { Years of } \\
\text { Education } \\
\text { M(SD) }\end{array}$ & $15.83(1.331)$ & $15(0.00)$ & $17.62(0.942)$ \\
\hline $\begin{array}{c}\text { Gender n (\%) } \\
\text { Female }\end{array}$ & $71(77.2)$ & $43(68.3)$ & $28(96.6)$ \\
\hline \multicolumn{3}{|c|}{ Clinical Characteristics } \\
\hline $\begin{array}{c}\text { Pittsburgh } \\
\text { Sleep Quality } \\
\text { Index M(SD) }\end{array}$ & $5.99(3.437)$ & $6.05(3.333)$ & $5.86(3.710)$ \\
\hline Optimism Index & 1 & 1 & 1 \\
\hline
\end{tabular}

Despite the success to find out the impact of insomnia on the five facets of optimism among young adults, there were certain limitations in the current study, which are as follows:

a) As the data collected was disproportionate in terms of sample size, gender therefore, standard generalization of the result is limited.

b) The optimism index scale questionnaire used in the study is under construction hence, superficial information regarding the facets of optimism was obtained which can be a factor that may affect the result analysis.

To overcome these shortcomings, further studies should be conducted so that a causal linkage between the variables can be drawn. Also, in order to give some more accurate result, different 
other aspects of the variable (e.g., stress and five facets of optimism; depression and optimism) should also be measured. Moreover, standardized objective instruments should be used to measure the variables, so that the reliability can be measured, and generalized results can be increased.

\section{Conclusion and Implication}

In summary, current study extends the knowledge towards the facets of optimism, elaborating that a better sleep quality is also related to higher level of positive facets viz. Positive Emotions, Engagement, Relationship, Meaningfulness \& Accomplishment. It also the expands the knowledge towards expansion of the facets of optimism, elaborating that a better sleep quality is related to five positive facets of optimism index scale viz. Positive Emotions, Engagement, Relationship, Meaningfulness \& Accomplishment. Future studies can make a greater effort in verifying the relationship among optimism, stress, depression, social support and how they interact with each other in order to predict insomnia or any sleep deprivation.

In a university setting, finding can be implemented to nurture optimism and guide sleep hygiene promotion and intervention among college students.

\section{Acknowledgement}

The authors would like to acknowledge all the participants of this study and also acknowledge all the participants who willingly participated in the study. Authors would also like to extend the gratitude toward Chancellor and Pro-vice chancellor of Amity University Haryana for their valuable support, motivation, and help during data collection and preparation of the manuscript.

\section{Refernces}

1. John U, Meyer C, Rumpf HJ, Hapke U (2005) Relationships of psychiatric disorders with sleep duration in an adult general population sample. J Psychiat Res 39: 577-583.

2. Meisinger C, Heier M, Löwel H (2007) Sleep duration and sleep complaints and risk of myocardial infarction in middle-aged men and women from the general population: the monica/kora augsburg cohort study. Sleep 30: 1121-1127.

3. Lemola S, Räikkönen K, Scheier MF (2011) Sleep quantity, quality and optimism in children. J Sleep Res 20: 12-20.

4. Sadeh A, Gruber R,Raviv A (2002) Sleep, neurobehavioral functioning, and behavior problems in school-age children. Child Dev 73: 405-417.

5. Gregory AM, Rijsdijk F, Lau JYF, Dahl RE, Eley TC (2009) The direction of longitudinal associations between sleep problems and depression symptoms: a study of twins aged 8 and 10 years. Sleep 32: 189-199.

6. Dahl RE, Harvey AG (2007) Sleep in children and adolescents with behavioral and emotional disorders. Sleep Med Clin 2: 501-511.

7. Alfano CA, Beidel DC, Turner SM, Lewin DS (2006) Preliminary evidence for sleep complaints among children referred for anxiety. Sleep Med 7: 467-473.

8. Soffer-Dudek, N, Sadeh, A, Dahl, RE, Rosenblat Stein S (2011) Poor sleep quality predicts deficient emotion information processing over time in early adolescence. Sleep 34: 1499-1508.

9. Benca RM, Cirelli C, Rattenborg NC, Tononi, G (2004) Basic science of sleep. In Comprehensive textbook of psychiatry. Lippincott, Williams and Williams pp. 280-294.
10. Rajmohan V, Mohandas E (2007) The limbic system. Indian journal of psychiatry 49(2): pp132.

11. Spielman AJ, Yang, CM, Glovinsky PB (2011) Sleep restriction therapy. In Behavioral Treatments for Sleep Disorders, Academic Press pp. 9-19.

12. Bootzin RR (1972) Stimulus control treatment for insomnia. Proceedings of the American Psychological Association 7: 395-396.

13. Harvey AG (2002) A cognitive model of insomnia. Behaviour research and therapy 40(8): 869-893.

14. Espie CA, Broomfield NM, MacMahon KM, Macphee LM, Taylor LM (2006) The attention-intention-effort pathway in the development of psychophysiologic insomnia: a theoretical review. Sleep medicine reviews, 10(4): 215-245.

15. Doghramji K (2006) The epidemiology and diagnosis of insomnia. American Journal of Managed Care 12(8): S214.

16. National Sleep Foundation (2002) Executive summary of the Sleep in America Poll.

17. Fredrickson PA, Krueger BR (1994) Insomnia associated with specific polysomnographic findings. Principles and practice of sleep medicine pp. 523-534.

18. Radler BT, Ryff CD (2010) Who participates? Accounting for longitudinal retention in the MIDUS National Study of Well-being. J Aging Health $22: 307-331$

19. Buysse DJ, Reynolds III, CF, Monk TH, Berman SR, Kupfer DJ (1989) The Pittsburgh Sleep Quality Index: a new instrument for psychiatric practice and research. Psychiatry research 28(2): 193-213.

20. Banerjee P, Yadav S, Puri A (2016) Construction of a Test on Optimism Index: Persoma ${ }^{\mathrm{TM}}$ Perspective. J Psychol Clin Psychiatry 5(6): 00310.

21. Rasmussen A, Smith H, Keller AS (2007) Factor structure of PTSD symptoms among west and central African refugees. Journal of Traumatic Stress Official Publication of The International Society for Traumatic Stress Studies 20(3): 271-280.

22. Giltay EJ, Geleijnse JM, Zitman FG, Hoekstra T, Schouten EG (2004) Dispositional optimism and all-cause and cardiovascular mortality in a prospective cohort of elderly Dutch men and women. Archives of general psychiatry 61(11): 1126-1135.

23. Cable N, Chandola T, Aida J, Sekine M, Netuveli G (2017) Can sleep disturbance influence changes in mental health status? Longitudinal research evidence from ageing studies in England and Japan. Sleep medicine 30: 216-221.

24. Haack M, Mullington JM (2005) Sustained sleep restriction reduces emotional and physical well-being. Pain 119(1-3): 56-64.

25. Rasmussen P, Brassard P, Adser H, Pedersen MV, Leick L, et al. (2009) Evidence for a release of brain-derived neurotrophic factor from the brain during exercise. Experimental physiology 94(10): 1062-1069.

26. Lau EY, Hui CH, Cheung SF, Lam J (2015) Bidirectional relationship between sleep and optimism with depressive mood as a mediator a longitudinal study of Chinese working adults. Journal of psychosomatic research 79(5): 428-434.

27. Roberts RE, Roberts CR, Duong HT (2008) Chronic insomnia and its negative consequences for health and functioning of adolescents a 12-month prospective study. Journal of Adolescent Health 42(3): 294302.

28. Kalak N, Lemola S, Brand S, Holsboer-Trachsler E, Grob A (2014) Sleep duration and subjective psychological well-being in adolescence a longitudinal study in Switzerland and Norway. Neuropsychiatric disease and treatment 10: pp 1199. 
(c) (i) This work is licensed under Creative

To Submit Your Article Click Here: Submit Article

DOI: $10.32474 /$ SJO.2019.02.000139

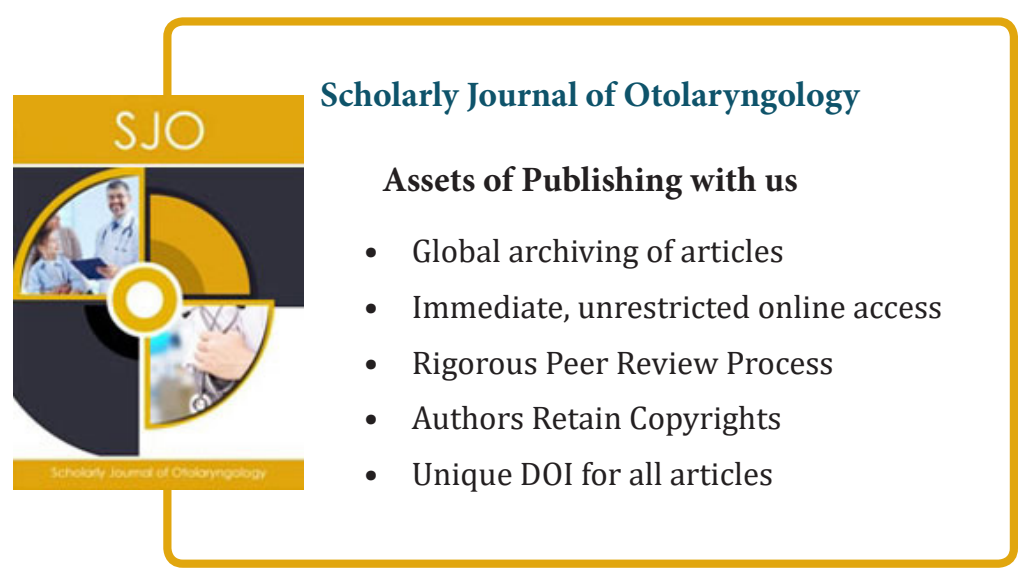

